\title{
Experiment Research on Hot-Rolling Processing of Nonsmooth Pit Surface
}

\author{
Yun-qing Gu, ${ }^{1}$ Tian-xing Fan, ${ }^{1}$ Jie-gang Mou, ${ }^{1}$ Wei-bo Yu, ${ }^{2}$ Gang Zhao, ${ }^{3}$ and Evan Wang ${ }^{4}$ \\ ${ }^{1}$ College of Mechanical Engineering, Zhejiang University of Technology, Hangzhou 310014, China \\ ${ }^{2}$ China Aviation Powerplant Research Institute, Zhuzhou 412002, China \\ ${ }^{3}$ College of Mechanical and Electrical Engineering, Harbin Engineering University, Harbin 150001, China \\ ${ }^{4}$ Institut National Polytechnique of Grenoble, Joseph Fourier University, Grenoble 38031, France \\ Correspondence should be addressed to Gang Zhao; zhaoheu@gmail.com
}

Received 8 November 2015; Accepted 18 January 2016

Academic Editor: Hoon Eui Jeong

Copyright (c) 2016 Yun-qing Gu et al. This is an open access article distributed under the Creative Commons Attribution License, which permits unrestricted use, distribution, and reproduction in any medium, provided the original work is properly cited.

\begin{abstract}
In order to achieve the nonsmooth surface drag reduction structure on the inner polymer coating of oil and gas pipelines and improve the efficiency of pipeline transport, a structural model of the machining robot on the pipe inner coating is established. Based on machining robot, an experimental technique is applied to research embossing and coating problems of rolling-head, and then the molding process rules under different conditions of rolling temperatures speeds and depth are analyzed. Also, an orthogonal experiment analysis method is employed to analyze the different effects of hot-rolling process apparatus on the embossed pits morphology and quality of rolling. The results also reveal that elevating the rolling temperature or decreasing the rolling speed can also improve the pit structure replication rates of the polymer coating surface, and the rolling feed has little effect on replication rates. After the rolling-head separates from the polymer coating, phenomenon of rebounding and refluxing of the polymer coating occurs, which is the reason of inability of the process. A continuous hot-rolling method for processing is used in the robot and the hot-rolling process of the processing apparatus is put in a dynamics analysis.
\end{abstract}

\section{Introduction}

Continuous hot-rolling transfer process employs rollers for rolling and continuous imprinting. Its characteristics include better flatness, smaller imprint pressure, and requiring simpler equipment; moreover, it needs only one mold to complete large-area substrate continuously imprinting, which can increase the production greatly [1]. Hot-rolling transfer processes include two copy types, the first uses a cylindrical mold with fine surface characteristics, and the second type uses roller with smooth cylindrical surface. In the first type, fine surface characteristics can be achieved by using the traditional microfabrication on its surface or by the method of covering smooth cylindrical surface with flexible membrane. The second type employs the traditional flatbed mold to achieve continuous transfer process. Regarding the exploration of this process, it is still in its infancy $[2,3]$.

Hot-rolling transfer processes include three types, the platen, stepping, and continuous rolling. For some advantages like better flatness, smaller imprint pressure, simple equipment, and large-area imprinting ability over the other processes, continuous rolling processes are preferred in gravure and flexible flexographic printing technology [4] and they adopt this roll-to-roll (R2R) method, which is applied to manufacture flexible electrophoresis display and has already been tested for its higher reliability and process performance [5]. In the actual production process, the typical R2R method consists of three steps, deposition, transfer, and packaging [6]. Tan et al. [1] firstly proposed and demonstrated one process which applied roller continuous manufacturing method to transfer technology in micro/nanomanufacturing area and presented the progress of roller nanoimprint lithography. Shen et al. [7] succeeded in producing 100 200 $\mu \mathrm{m}$ cylindrical microlens on the polycarbonate substrate by using the fine hot transfer process and studied the relationship between the lens size and imprinting pressure, temperature, and time during the microlens production process. Shan et al. $[8,9]$ successfully produced vertical microlenses and an optical conversion platform by using hot transfer 
technology. Their study showed that the embossed polycarbonate substrate temperature should be higher than $185^{\circ} \mathrm{C}$, and imprinting pressure should be greater than $1.75 \mathrm{~kg} / \mathrm{mm}^{2}$. Liang et al. [10] proposed a continuous process using rollto-roll method. This continuous roll-to-roll rolling transfer process can manufacture products such as flexible displays at low cost and high efficiency. Chang et al. [11] proposed a method which can use air pressure to make silicon mold during the hot transfer process, when the air pressure, temperature, and pressure holding time was $10 \sim 40 \mathrm{kgf} / \mathrm{cm}^{2}$, $150^{\circ} \mathrm{C}$, and $30 \sim 90 \mathrm{~s}$, respectively. This could produce neat uniform and focusing powerful microlens array. Charest et al. [12] proposed a method of using hot transfer process to produce the morphology structure on the polymer substrate for cell growth and osteoblast growth direction response along the surface. In order to improve the release properties of Ni substrate, Byeon et al. [13] placed a film of $\mathrm{SiO}_{2}$ on the surface of Ni substrate and then coated a film of SAM (self-assembled monolayer) on the surface of $\mathrm{SiO}_{2}$. He found that it was very easy for the $\mathrm{Ni}$ substrate to be released during the polymer hot transfer. Air bubbles defects are often encountered during the manufacturing process of polymer. To avoid them, Mekaru et al. [14] added an ultrasonic vibration in longitudinal direction in the hot transfer process. This method greatly minimized the bubble defects. Lee et al. [15] incorporated a tiny capillary electrophoresis device on the surface of polymethyl methacrylate to separate the DNA sequences. The trench which was produced has good repeatability, whose error was less than 1\%. Chang and Yang [16] proposed a method of using uniform pressure to load pressure, which can improve the accuracy of hot imprinting on the whole entire substrate. Wang et al. [17] developed a novel full-color electrophoretic membrane manufacturing process by using rolling method. An orthogonal test method was proposed by $\mathrm{Li}$ et al. [18] to search the relationship between hot transfer process conditions and transfer accuracy with imprinting temperature, pressure, and imprinting lead time. Experiment revealed that imprinting temperature and pressure determined the accuracy replication of fine groove depth; however, the accuracy replication of groove width and shape was determined by holding pressure and heating time. Lan et al. [19-21] considered the polymer material's rheological properties changes with temperature and established a noncrystalline polymer material viscoelastic model near glass transition temperature and also built a simulation model of platen hot transfer process; moreover, they analyzed the effect rules between the process parameters and quality of transfer. Finally, for the large-area continuous transfer problems of fine characteristic structure, they proposed an evaluating method for continuous roller hot transfer quality using transfer replication rate and explored the effect rules between rolling process parameters and forming quality, which provided a guidance to the fine hot transfer process design of noncrystalline polymer substrate.

In this paper, for the purpose of reducing resistance and energy of oil and gas pipelines, we present a manufacturing method for a nonsmooth surface reducing resistance structure on the surface of pipe inner-wall polymer coating.
Also, we establish a pipe inner-wall machining robot model and apply the pipe inner-wall machining robot as carrier to analyze the problem of hot-rolling-head coating. In addition, we research the effects between different process parameters and hot-rolling results.

\section{Modeling of Pipe Inner-Wall Machining Robot}

The main job of modeling pipe inner-wall machining robot is to achieve micromachined structures drag reduction on the inner polymer coating surface of the oil and gas pipelines. The model has the capacity of walking, positioning, and highprecision microsurface processing inside the pipe. Modular design concept is used in the design model of pipe innerwall machining robot in order to minimize the correlation between the various modules. According to its function, model of pipe inner-wall machining robot is divided into walking modules and processing modules. The walking modules are responsible for the entire robot to walk in the pipeline, and the processing module is responsible for roll processing the polymer coating on the surface of the pipe wall.

2.1. Modeling of Walking Modules. The structure of walking equipment is as follows [22]: (1) linear motors, (2) screw nut, (3) screw, (4) support module, (5) stepper motor, (6) adjusting screw, (7) walking wheel, and (8) adjusting nut.

The required parameters of the walking equipment for pipe inner-wall machining robot are as follows: (1) powerful load capacity provides greater traction, (2) good stability, and (3) easy and simple control to achieve precise positioning. The equipment is peristaltic walkway, which can be divided into five parts, such as diameter adapting wheel mechanism 1, front support mechanism, peristaltic walk mechanism, back support mechanism, and diameter adapting wheel mechanism 2 .

In the walking equipment, diameter adapting mechanism 1 and front supporting mechanism constitute peristaltic front joint, diameter adapting mechanism 2 and back supporting mechanism constitute peristaltic back joint, and the equipment of peristaltic walk mechanism is in the middle of front and back joints. During the peristaltic walking, linear motors move to cause the elongation and contraction of the driving front and back joint, and each mechanism unites to achieve the robot's walking function. The work steps of walking equipment are as follows. (1) Front support loosens followed by the clamping of the back support, and then linear motors elongation drives front joint to move forward. (2) Front support clamps, and back support loosens, and then linear motors contraction drives the back joint to move forward. (3) Repeat the previous two steps actions to achieve a peristaltic walking.

Diameter adapting wheel mechanism is a hand tightening structure. Before machining the robot on the pipe innerwall, manually adjusting the diameter wheel mechanism nut presses the nut against the pipe inner-wall. Front and back supporting mechanism is slider-crank mechanism under the control of screw nut. In the process of working, the driving 
motor drives the screw nut to make linear slide, and screw nut drives the crank to make swinging movement to achieve the support module' pitching movement, which can achieve the support module clamping and releasing action in the pipe inner-wall. Diameter adapting wheel mechanism and supporting mechanism are both used symmetrically in the distributed architecture. It means that diameter adapting wheel mechanism has four groups for walking wheel structure with uniform distribution of $90^{\circ}$ up, and supporting mechanism also has four groups to support modules with uniform distribution of $90^{\circ}$ up. Diameter adapting wheel mechanism and crank slider support mechanism are both applied as truss structures, which possess powerful accommodating ability in pipe inner-wall, so in a certain range of pipe inner-wall, this walking equipment can adjust its truss swing angle to adapt to those changes of pipe inner-wall.

2.2. Modeling of Processing Module. The structure of processing equipment is as follows [22]: (1) DC servo motor, (2) driven bevel gear, (3) thrust ball bearing, (4) transmission shaft, (5) axle drive bevel pinion, (6) rolling tool, (7) heating rod, (8) slideway, (9) outer sleeve, (10) cam, (11) idler wheel, (12) cam, (13) outer sleeve, and (14) hollow shaft.

Processing module is on the back of walking equipment and constitutes one part of walking equipment; the walking equipment can drag it to the processing position. This equipment includes three parts: front processing components, driving components, and back processing components.

Driving components consist of DC servo motor and a series of bevel gears. Bevel gear system is a master-slave structure, which means a driving wheel drives two driven wheels reverse synchronous rotation. Front processing components and back processing components have the same symmetrical arrangement structure. The main parts of processing components are knife-head mechanism, slide track orienting mechanism, cam radial drive mechanism, and the inner and outer sleeve circumferential rotating mechanism. The distance of roll cutter is $190 \mathrm{~mm}$ between the two processing components. Processing components can complete two discontinuous processing on the polymer coating at one running. The length processed is $190 \mathrm{~mm}$, which needs the back processing components to make two consecutive forward processing complete.

In the processing of microdrag reduction surface structures, roll cutter rolled around the pipe axis suffers tangential reaction force, which passes to the processing component body at last, causing the body's torsion deformation. It not only affects the structure's stability, but also affects the pit's direction processing and eventually leads to drag reduction structure which cannot achieve the desired drag reduction effect. In order to solve this problem, we use the method of dual tool counterrotating in the design. Transmission of bevel gear train can achieve synchronous reverse rotation of the left and right bevel gears connecting the cutter sets with the left and right bevel gears to achieve synchronous reverse rotation of two cutter sets. Under this processing mode, the force of tangential reaction force given to processing apparatus body can cancel each other and attains the equilibrium condition. During the processing, these two sets cutters rotate with

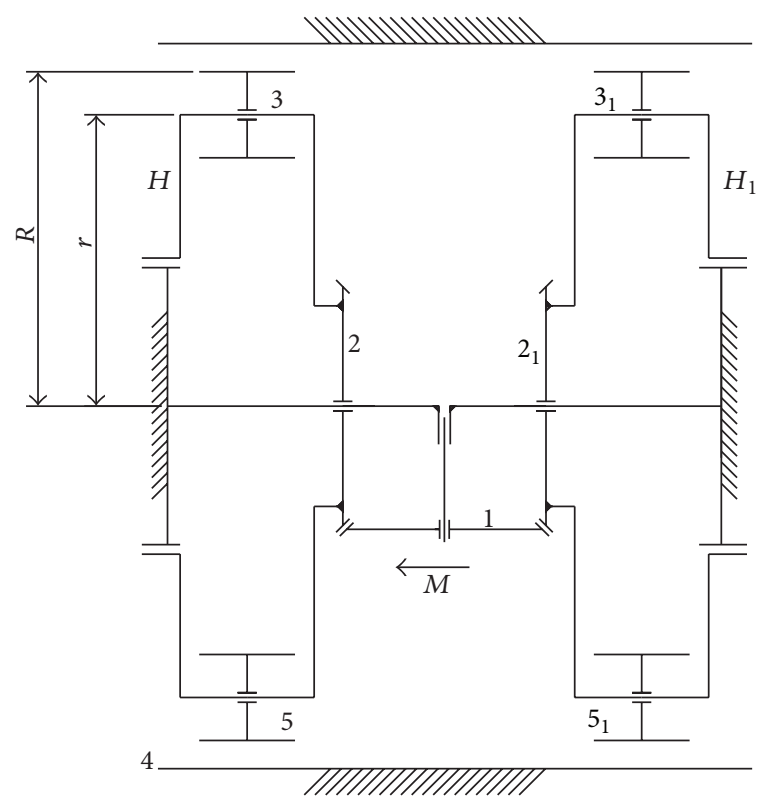

Figure 1: The model of processing module gear drive system.

left and right bevel gears, respectively, achieving the main movement. Under the role of cam acting and slide guiding, radial turret stretched along radial direction; it can achieve the cutters feed and retract movements in the processing. This structure enables the target single-power multifunction. It means one drive motor drives the entire process components to achieve the cutter's main movement, feed movement, and retract movement, making the structure more compact with better action coordinating in the processing.

\section{Analysis of Hot-Rolling Machining Robot}

Hot-rolling-head is the main component of the pipe innerwall machining robot, whose processing performance has a direct impact on the quality of the polymer coating surface.

3.1. Dynamics Analysis of Hot-Rolling Processing. During the processing of the pipe inner-wall machining robot, the rolling cutter's rolling movement on the pipe polymer coating surface can be simplified to an inner gear and an outer gear meshing process. The pipe polymer coating is equivalent to the inner gear, and rolling cutter is equivalent to the outer gear. The model of simplified processing module gear drive system is shown as in Figure 1.

Figure 1 shows that, on the processing module, gear 1 is the main driving bevel gear and is connected with drive motor, gear 2 is the left driven bevel gear, gear $2_{1}$ is the right driven bevel gear, $H$ is the front processing component of the outer sleeve, $H_{1}$ is back processing component of the outer sleeve, gear 3 , gear $3_{1}$, gear 5 , and gear $5_{1}$ are four roll cutters of the processing modules, and 4 is pipe inner-wall polymer coating.

As the front and back processing component's structure and movement are the same, it can be simplified in the dynamics analysis, only analysis of the front processing 
component. At the same time, as 3 and 5 and $3_{1}$ and $5_{1}$ are both installed along the centerline symmetrically, and in the processing, one gravity has always done positive work, gravity has always done negative work, and their absolute values are equal; in the whole processing, 3 and 5 and $3_{1}$ and $5_{1}$ are both zero gravity acting, so gravity is not considered in the analysis.

In a system of particles with ideal constraints, in any instant movement, all the active forces system, and inertial force system, the sum of the elementary work is zero in any virtual displacements; namely,

$$
\sum_{i=1}^{n}\left(\overrightarrow{F_{i}}-m_{i} \overrightarrow{a_{i}}\right) \overrightarrow{\delta_{r_{i}}}=0
$$

If moment situation is considered, kinetic equation can be expressed as

$$
\sum\left(M_{i} \delta_{\varphi_{i}}-m_{i} \overrightarrow{a_{i}} \overrightarrow{\delta_{r_{i}}}\right)=0
$$

From (1) and (2) we can get gear train transmission mechanism for kinetic equation as follows:

$$
\begin{aligned}
& \frac{1}{2} M \dot{\varphi}_{1}-J_{1} \ddot{\varphi}_{1} \dot{\varphi}_{1}-\frac{1}{\eta_{1}}\left(J_{2}+J_{H}\right) \ddot{\varphi}_{H} \dot{\varphi}_{H} \\
& \quad-\frac{1}{\eta_{1} \eta_{2}}\left(J_{3} \ddot{\varphi}_{3} \dot{\varphi}_{3}+J_{5} \ddot{\varphi}_{5} \dot{\varphi}_{5}\right) \\
& \quad-\frac{1}{\eta_{1}} r^{2}\left(m_{3}+m_{5}\right) \ddot{\varphi}_{H} \dot{\varphi}_{H}-\frac{1}{\eta_{1} \eta_{2}} F^{\prime} \dot{\varphi}_{3}(R-r) \\
& =0
\end{aligned}
$$

where $J_{1}, J_{2}, J_{3}, J_{5}$, and $J_{H}$ are driving bevel gear 1 , driven bevel gear 2 , gear 3 , gear 5 , and $H$ carrier of inertia. $m_{3}$ and $m_{5}$ are the quality of gear 3 and gear 5. $\dot{\varphi}_{1}, \dot{\varphi}_{3}, \dot{\varphi}_{5}$, and $\dot{\varphi}_{H}$ are the angular velocity of driven bevel gear 1, gear 3, gear 5, and carrier H. $\ddot{\varphi}_{1}, \ddot{\varphi}_{3}, \ddot{\varphi}_{5}$, and $\ddot{\varphi}_{H}$ are the angular acceleration of driving bevel gear 1 , gear 3 , gear 5 , and carrier $H . F^{\prime}$ is the reaction force in the processing of roll cutter. $\eta_{1}$ is the transmission efficiency between the outer sleeve rotating system from driving bevel gear and driven bevel gear. $\eta_{2}$ is the transmission efficiency of roll cutter rotating mechanism. $M$ is the output torque of drive motor.

From $i_{3 H}=\dot{\varphi}_{3} / \dot{\varphi}_{H}=\ddot{\varphi}_{3} / \ddot{\varphi}_{H}=-r /(R-r)$ the following can be obtained:

$$
\begin{aligned}
& \dot{\varphi}_{3}=-\frac{r}{R-r} \dot{\varphi}_{H}, \\
& \ddot{\varphi}_{3}=-\frac{r}{R-r} \ddot{\varphi}_{H} .
\end{aligned}
$$

Gear 3's structure and motion parameters are all the same as gear 5 , so

$$
\begin{aligned}
& m_{3}=m_{5}, \\
& \dot{\varphi}_{3}=\dot{\varphi}_{5}, \\
& \ddot{\varphi}_{3}=\ddot{\varphi}_{5} .
\end{aligned}
$$

By putting (4), (5), and the following equation $i_{1 H}=i_{12}=$ $\dot{\varphi}_{1} / \dot{\varphi}_{H}=\ddot{\varphi}_{1} / \ddot{\varphi}_{H} i=-z_{2} / z_{1}$ in (3), then we can get the kinetic equation of roll cutter (gear 3 ) as follows:

$$
\ddot{\varphi}_{3}=\frac{(R-r) r z_{1}\left(\eta_{1} \eta_{2} z_{2} M-2 z_{1} r F^{\prime}\right)}{2\left[z_{1}^{2}(R-r)^{2} \eta_{2}\left(J_{2}+J_{H}-2 m_{3} r^{2}\right)+\eta_{1} \eta_{2} z_{2}^{2} J_{1}(R-r)^{2}+2 z_{1}^{2} J_{3} r^{2}\right]} .
$$

Equation (6) provides the gear train transmission dynamics expression of the pipe inner-wall machining robot processing module. This formula shows the relationship between angular acceleration $\ddot{\varphi}_{3}$ of roll cutter and the output torque $M$ of drive motor and reaction force $F^{\prime}$. Each component of the processing module is designed by considering the basics of the roll processing parameters, according to this relationship.

3.2. Analysis of Hot-Rolling-Head and Its Power. The abovementioned paragraph analyzes the overall structure of pipe inner-wall machining robot. The main function of the machining robot is to apply a vector for hot-rolling-head, and the key core technology lies in how to roll out a nonsmooth surface structure with drag reduction effect, using hot-rolling-head to press on the surface of pipe innerwall polymer coating.

3.2.1. Surface Morphology of Rolling-Head. The effect of drag reduction [23-26] on nonsmooth surface has already been recognized by domestic and foreign researchers; its main types are pit-type, scales-type, and triangular groove-type [27-31]. The structure size of pit-type is two magnitudes bigger than triangular groove-type, and scales-type usually uses the method of biological replicates to produce. The production costs are high, and it is difficult to be applied on large scale $[32,33]$. Considering the advantages of processing and maintenance and combining the actual situation of pipe wall machining robot, select the convex hull as the surface morphology of the rolling-head, and then achieve to roll out the pits characteristic structure on polymer coating surface.

The rolling-head's radius $R$ is $27 \mathrm{~mm}$, and length $L$ is $90 \mathrm{~mm}$. There is a through hole whose diameter is $36 \mathrm{~mm}$ in the inner rolling-head for placement of heating rods into it. Convex hull is hemispherical of radius $r=0.8 \mathrm{~mm}$ and length $h=0.8 \mathrm{~mm}$, the convex hull is on the rolling-head and arranged in a diamond structure, and its axial spacing $s=16 \mathrm{~mm}$ and circumferential angle $\theta=20^{\circ}$. The model of hot-rolling-head is showed as in Figure 2. 


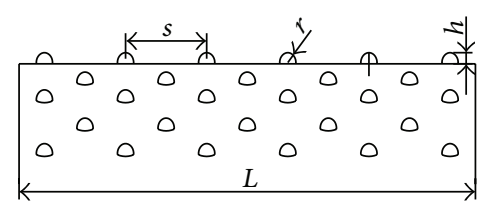

(a)

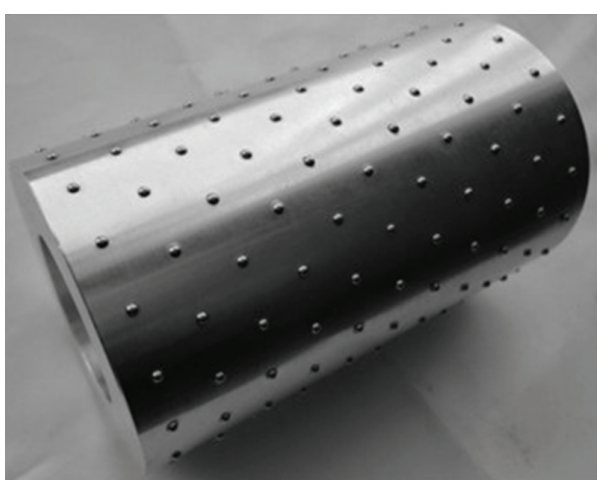

(b)

FIGURE 2: Model of hot-rolling-head: (a) the morphology of rolling-head parameter and (b) the physical hot-rolling-head.

3.2.2. Power Analysis of Hot-Rolling-Head. The heat requirement of the hot-rolling-head is start-up and holding temperature power. Start-up power $W_{q}$ is used for heating roller to change room temperature $T_{1}$ to the required temperature $T_{2}$. $W_{q}$ is composed of heating power and distribute power.

Heating power $W_{1}$ is the power required to heat the hotrolling's temperature from $T_{1}$ to the required temperature $T_{2}$, so formula $W_{1}$ is

$$
W_{1}=\frac{C M\left(T_{2}-T_{1}\right)}{t},
$$

where $C$ is the specific heat of hot rolling, $M$ is the quality of hot rolling, and $t$ is the heating time.

Distributing power is the power which hot-rolling lost during the temperature elevation because of conduction, radiation, and convection. Because the hot-rolling-head's temperature always changes during heating time, it is difficult to compute distributed power theoretically. Therefore, the power of required temperature $T_{2}$ multiplied by thermal coefficient $K$ constitutes the distribute power, as is shown in

$$
W_{2}=K W_{c} \text {, }
$$

where $W_{c}$ is holding temperature power and $K$ is thermal coefficient.

Holding temperature power $W_{c}$ is the power to hold the hot-rolling-head's temperature without changing, it is constituted by heat conduction power $W_{d}$, heat radiated power $W_{f}$, and heat convection power $W_{l}$, and its formula is

$$
W_{c}=W_{d}+W_{f}+W_{l}
$$

The formula of heat conduction power $W_{d}$ is

$$
W_{d}=\sum \frac{\Delta T A \lambda}{\delta}
$$

where $\Delta T$ is the temperature difference between hot rollers and polymer coating, $A$ is the contact area between hot rollers and polymer coating, $\lambda$ is the thermal conductivity, and $\delta$ is the roll head thickness.
The formula of heat radiated power $W_{f}$ is

$$
W_{f}=\varepsilon q_{h} A_{1}
$$

where $A_{1}$ is the remaining hot-roller surface area which is contacted with the polymer coating, $\varepsilon$ is the radiation coefficient of hot roller, and $q_{h}$ is the heat radiation loss per unit area.

The formula of heat convection power $W_{l}$ is

$$
W_{l}=\eta \alpha_{1} A_{1}
$$

where $a_{1}$ is convective heat loss dissipation factor and $\eta$ is convective heat loss correction factor.

The formula of holding temperature power $W_{c}$ is

$$
W_{c}=\sum \frac{\Delta T A \lambda}{\delta}+\varepsilon q_{h} A_{1}+\eta \alpha_{1} A_{1}
$$

The formula of starting power is

$$
\begin{aligned}
W_{q}= & \frac{C M\left(T_{2}-T_{1}\right)}{t} \\
& +K\left(\sum \frac{\Delta T A \lambda}{\delta}+\varepsilon q_{h} A_{1}+\eta \alpha_{1} A_{1}\right) .
\end{aligned}
$$

Combined with the hot roller's specific circumstances of pipe wall polymer coating, $T_{1}=18^{\circ} \mathrm{C}, T_{2}=140^{\circ} \mathrm{C}, \mathrm{C}=$ $0.88 \mathrm{KJ} /\left(\mathrm{kg} \cdot{ }^{\circ} \mathrm{C}\right), M=0.41 \mathrm{~kg}, K=0.55 \sim 0.65, A=3 \mathrm{~cm}^{2}$, $A_{1}=136 \mathrm{~cm}^{2}, a_{1}=0.124 \mathrm{~W} / \mathrm{cm}^{2}, \eta=1, \lambda=$ $0.001 \mathrm{~W} /\left(\mathrm{cm} \cdot{ }^{\circ} \mathrm{C}\right), W_{c}=30 \mathrm{~W}$, and $W_{q}=210 \mathrm{~W}$ are determined. Considering the complexity during the actual processing on pipe wall polymer coating, the selected power should be greater than theoretical value. At last, the required heating power is $300 \mathrm{~W}$.

Based on the temperature sensor characteristics, select two angles to choose the temperature sensor. One is raising the temperature measurement accuracy, and another is reducing the temperature measurement error. Choosing thermocouple as temperature sensor, the measurement error of thermocouple is smaller than measurement error of thermal resistance. The hot-rolling-head's temperature measurement 
range is $0 \sim 500^{\circ} \mathrm{C}$ and its display error does not exceed $0.5 \%$. So relay output is selected, which could receive input thermocouple temperature sensor, and working environment is the oil and gas pipelines. Digital temperature control device is used.

\section{Text Set and Test Method}

Hot-rolling crafts of pipe inner-wall machining robot are a continuous replication process, and its continuous replication process can also be applied to copy the characteristic structure of nonsmooth convex hull which has drag reduction effect on roller surface to the polymer coating surface in order to achieve a large-area nonsmooth surface replication. Based on the above theory, machining robot is built on the pipe inner-wall, and then the hot-rolling process is studied. Under different roll temperature, speed, and depth, the temperature and time dependence of the polymer material are analyzed.

4.1. Prototype Debugging. Based on the above analysis, a test prototype of pipe inner-wall machining robot is built. After the assembly manufacturing of machining robot parts followed by prototype assembling, prototype debugging is needed to verify the control reliability of the motor and rationality of control strategy. Machining robot's debugging work required the drive motor to achieve the required parameters. Machining robot walking modules have 57H56 DC stepper motor and TG-300-D motorized faders, machining robot processing module has 90BLDC servo motor, and the debugging contains hardware, software, and system debugging.

Hardware debugging contains power test, I/O connection check, and safety circuit confirmation. For programming system, we use software STEP7. First, we achieve the interface between the computer and the PLC and ensure proper communication between the two. Then, we download the system to the PLC and open the actual operation of the online monitoring. At last, we compared the PLC actual data with the project data and made proper adjustments. System debugging is the whole debugging between control systems and pipe inner-wall machining robot. During the debugging, the rule is to meet the control requirements and ensure system security. In order to select the optimal control parameters, first, we let the robot walk in the pipeline, and then, according to the pipeline diameter and walking displacement, we adjust the pulse volume and analog observation.

4.2. Coating of Polymer Pipe Inner-Wall. The main job of inner-wall pipelines coating is to protect oil and gas pipelines against transporting media's corrosion and to reduce the resistance of transporting media. Gas medium is a combination of a mixture of combustible and noncombustible material, whose main component is a substance of low molecular weight saturated with hydrocarbons, and some small amounts of nonhydrocarbon substances $[34,35]$. Since some acidic substance constitutes the medium, and it can corrode the inner-wall, a polyurethane category test is selected at room temperature for curable coating taking requirements of oil and gas pipelines drag-reducing into account. This coating is composed of polyisocyanate prepolymer, fillers, additives, solvents, and some other materials. And it has good wear resistance and good adhesion, with good water resistance, moisture resistance, oil resistance, good acid and alkali resistance, solvent resistance, and chemical resistance, so it is very good for the drag reduction and corrosion on pipe coating equipment.

The process of polymer coating is as follows:

(1) Cleaning of pipe inner-wall: the dirt and other attachments are wiped on the pipe inner-wall by using mechanical cleaning methods, solvents or highpressure water, and so forth. Then the pipeline is preheated to temperature $3^{\circ} \mathrm{C}$ higher than the room temperature, and shot blasting is applied to do the rust treatment to achieve the Sa2.5 class. Finally compressed air is employed for purging the sand and dust off the pipe inner-wall.

(2) Coating of pipe inner-wall: the inner-wall is coated manually in order to maintain the coating thickness uniformity; multiple coatings are desirable with a brush whose thickness is determined by testing requirements. Finally, the paint is sprayed to the pipe inner-wall by an airless spray pump.

(3) The handle of coating solidification: the coating solidification usually relies on solvent evaporation or coating chemical reaction in terms of the actual situation of pipeline, but we use the hot air to make coating solidification faster.

4.3. Design Conditions of Hot-Rolling Process. The hot-rolling process is widely used in the field of micromanufacturing as a manufacturing technique. However, in order to achieve largearea nonsmooth surface replication molding technology as a continuous rolling, its process control parameters are still in the research and practice phases. The main affecting factors of hot-rolling process parameters are temperature, time, speed, and pressure. Since the rolling-head of machining robot uses the cam's lift and return to achieve the rolling-head for carrying out the plate polymer coating, different temperature, rolling speed, rolling time, and rolling are mainly considered for analysis of the effect of rolling.

Hot-rolling process mainly includes three phases: the preheating, hot-rolling, and cooling. The temperature curve versus time in the process is showed as in Figure 3. Specific steps are as follows: in the phases of preheating, the rollinghead's temperature is raised to the temperature of polymer glass transition temperature above $T_{1}$, then maintaining this temperature for a while $S T_{1}$. The polymer's state is viscoelastic state at this temperature. In the phases of hot rolling, to roll the pipeline polymer coating by cam's lifting for a rolling depth $h$ and keep the rolling time for a while $h T_{1}$, the pressure of coating surface becomes smaller as the temperature of the polymer coating decreases at this time, and the temperature elevates through the conduction heating between hot rollers and coating. High temperature is maintained in some localized parts of the coating surface only, to ensure a faster mobility, and the bottom's temperature is low enough, so there is no mobility produced on the bottom of coating. In 


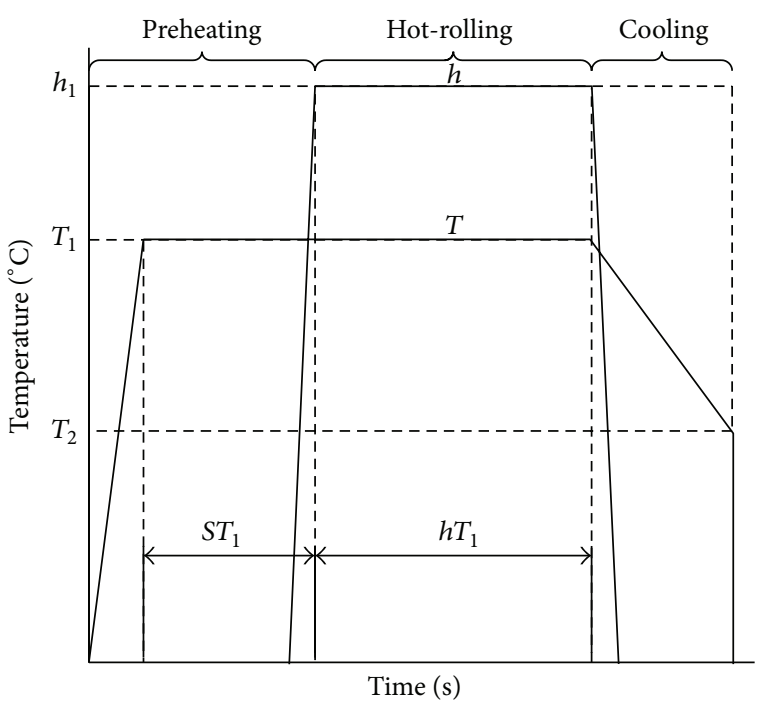

FIGURE 3: Curve of temperature versus that in hot-rolling process.

the hot cooling phase, the temperature of rolling-head and polymer pipe inner-wall is lowered to the glass transition temperature below $T_{2}$ instantaneously. Finally, the coating is cooled in the natural environment, and its temperature slowly returned to normal; in addition, rolling-head separates from the polymer coating on the cam's return.

4.4. Replication Rate and Measurement. In order to evaluate replication results under different hot-rolling process parameters, we define the replication rate as the final pits structure characteristics of the coating surface, which means that the replication rate is the volume ratio between all pit features and standard pit features after the forming rolling. It is very difficult to accurately measure and calculate pits structure characteristics, so the laser displacement scanner (type FT5070F) is used to measure sectional shape of pits structure characteristics on polymer coating. This can change the replication rate to area ratio between the intermediate sectional area of pits structure characteristics on polymer coating and the intermediate sectional area of standard pits structure characteristic:

$$
\eta=\frac{S^{\prime}}{S} \times 100 \%
$$

where $S^{\prime}$ is the intermediate sectional area of pits structure characteristics on polymer coating and $S$ is the intermediate sectional area of standard pits structure characteristic.

The detection distance of laser displacement scanner is $30 \sim 100 \mathrm{~mm}$, and its accuracy is $0.03 \sim 0.1 \mathrm{~mm}(0.1 \%$ of MBE). Light source is red laser $650 \mathrm{~nm}$, and working temperature is $-10 \sim 60^{\circ} \mathrm{C}$. This scanner is connected to the computer through the serial port and got the data acquisition by serial debugging software, and this scanner calculated the replication rate by measuring the sectional shape of rolling pits structure characteristics in processing. The distance between test pits and reference points is measured by laser displacement scanner and the output is communicated with

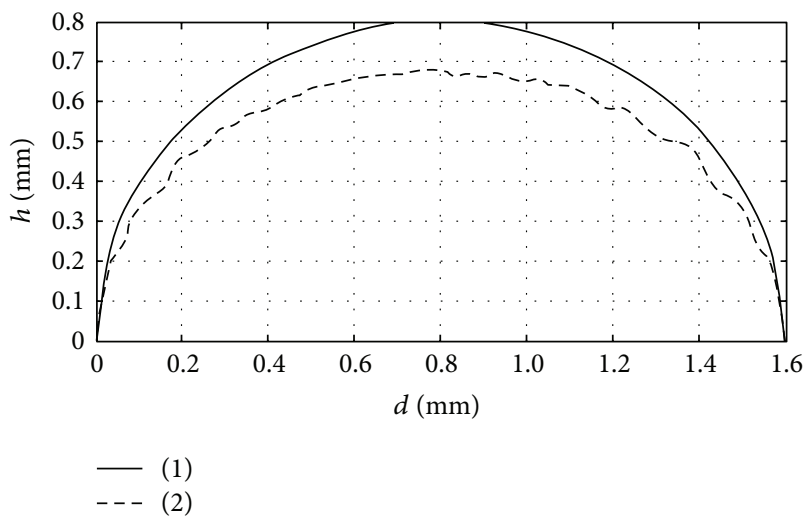

FIgURE 4: The comparison chart between actual intermediate sectional area and ideal intermediate sectional area: (1) ideal pit morphology and (2) actual pit morphology.

the computer. Also, the measured data is processed by MATLAB software, and it restores the sectional shape of rolling characteristic structure and then calculates the sectional area by the method of trapz function.

\section{Test Results and Analysis}

Control parameters in the hot-rolling process are as follows: the rolling temperature is $150^{\circ} \mathrm{C}$, rolling depth is $0.8 \mathrm{~mm}$, and rolling speed is $1 \mathrm{rad} / \mathrm{s}$. The formed pits sectional morphologies are shown in Figure 4. It compares the characteristic structure of hot-rolling-head with the pits characteristic structure of polymer coating. It can be inferred that pits structure replication can be achieved on polymer coating, and consistent lines morphology can be achieved with the standard pits. Through formula (15), we calculate that the replication rate is $85.0 \%$, which means that the replication rate is higher enough, and it also can verify the design rationality and the processing accuracy of the pipe inner-wall machining robot.

In order to study the effect of the rolling process parameters on replication, different rolling depth $h$, rolling speed $\omega$, and rolling temperature $T$ are chosen to have a series of tests. Under the condition of $h=0.2 \mathrm{~mm}, T=140^{\circ} \mathrm{C}, 145^{\circ} \mathrm{C}, 150^{\circ} \mathrm{C}$, $\omega=0.5 \mathrm{rad} / \mathrm{s}$, and $1 \mathrm{rad} / \mathrm{s}, 1.5 \mathrm{rad} / \mathrm{s}$, we start a rolling test and measure and calculate the hot-rolling replication rate. After that, we develop a variable chart for varying replication rate and rolling temperature, as is shown in Figure 5.

The histogram in Figure 5 shows that under the same rolling depth, replication rate increases at elevated temperature, and at constant temperature, replication rate increases at lowered rolling speed. The polymer is very sensitive to temperature changing, so its mechanical properties is viscoelastic above the glass transition temperature, and its stress relaxation speed also increases, as its creep speed increases with increasing temperature. When the rollinghead is processed on the polymer coating, elastic deformation occurred firstly, followed by viscous deformation; viscous deformation is much bigger than elastic deformation. At the end time of $T=140^{\circ} \mathrm{C}, 145^{\circ} \mathrm{C}$, and $150^{\circ} \mathrm{C}$, there is a 


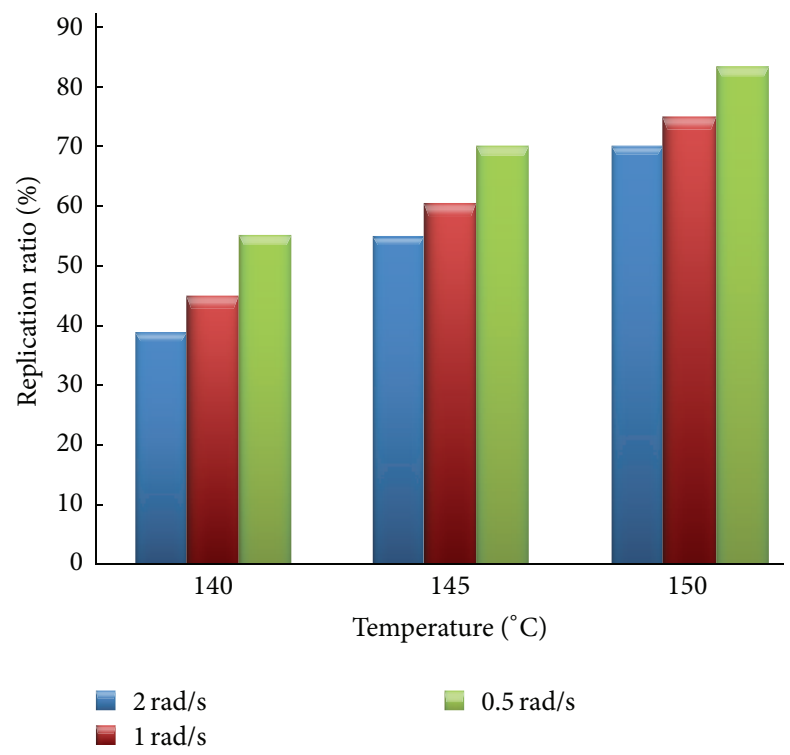

FIGURE 5: The changing chart in which replication rate changed with rolling temperature.

proper amount of rebound phenomena of the pit morphology on polymer coating, due to the elastic deformation when hot-rolling-head separates from polymer coating. When the polymer coating temperature is lowered below the glass transition temperature, its mechanical state is glassy, and the rebound phenomenon stops. So the accuracy of replication can be improved by increasing temperature or decreasing rolling speed in the actual process.

Under the circumstance of $T=150^{\circ} \mathrm{C}, h=0.2 \mathrm{~mm}$, $0.4 \mathrm{~mm}, 0.6 \mathrm{~mm}, \omega=0.5 \mathrm{rad} / \mathrm{s}, 1 \mathrm{rad} / \mathrm{s}$, and $1.5 \mathrm{rad} / \mathrm{s}$ another rolling test is conducted and then measured and calculated the hot-rolling replication rate. We develop another chart in which replication rate changed with rolling depth, as is showed in Figure 6. As it can be seen from the histogram in Figure 6, replication rate increases with decreasing rolling speed, which shows good time-dependency of polymer coating, under the same rolling depth. Replication rate increases with increasing rolling depth at low rolling speed, with obvious change of replication rate. At higher rolling speed, replication rate increases slightly with the changing rolling depth. It shows that increase of the rolling depth cannot increase the replication accuracy significantly.

In order to elaborate further the effect of the three main control parameters on replication rate in hot-rolling process, the orthogonal method is used to analyze the parameters in hot-rolling process. Factor $A$ is rolling depth, factor $B$ is rolling speed, and factor $C$ is rolling temperature, as shown in Table 1.

In Table 1, three factors and three horizontal test tables are created with a total of $3^{3}=27$ times according to $L_{9}\left(3^{4}\right)$, selected 9 from 27 , and those selected numbers are 1, 5, 9, 11, 15, $16,21,22$, and 26 , and then test those selected numbers under new ID numbers 1 to 9 . In experimental tests, $1 / 3$ of total test numbers are done. In order to ensure the stability of the test results, the tests are repeated three times in each case, and

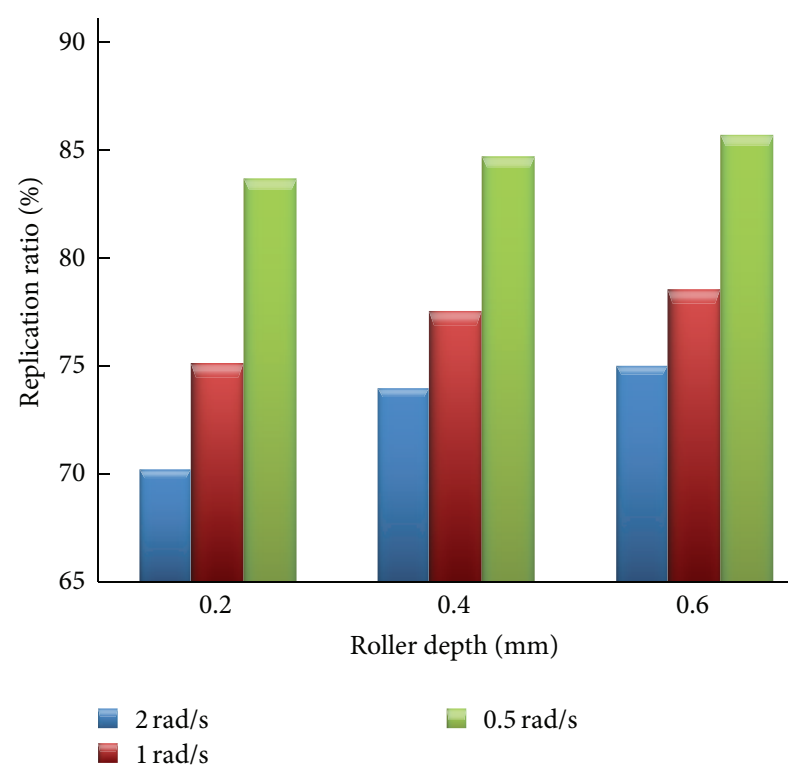

FIGURE 6: A changing chart in which replication rate changed with rolling depth.

TABLE 1: Three factors and 3 horizontal test tables.

\begin{tabular}{lccc}
\hline Horizontal & \multicolumn{3}{c}{ Factors } \\
& $\begin{array}{c}A \\
h / \mathrm{mm}\end{array}$ & $\begin{array}{c}B \\
\omega /\left(\mathrm{rad} \cdot \mathrm{s}^{-1}\right)\end{array}$ & $\begin{array}{c}C \\
T{ }^{\circ} \mathrm{C}\end{array}$ \\
\hline 1 & 0.2 & 2 & 140 \\
2 & 0.4 & 1 & 145 \\
3 & 0.6 & 0.5 & 150 \\
\hline
\end{tabular}

then we get three factors coupling test results $A, B$, and $C$, as shown in Table 2 . In Table $2, H_{1}$ represents the total average values of replication rate in order factors $A, B$, and $C$ in the first level of the test; $H_{2}$ represents the total average values of replication rate in order factors $A, B$, and $C$ in the second level of the test; $H_{3}$ represents the total average values of replication rate in order factors $A, B$, and $C$ in the third level of the test. So $h_{1}=H_{1} / 3, h_{2}=H_{2} / 3$, and $h_{3}=H_{3} / 3$ are the average value of each corresponding level. In the same column, the maximum value in $h_{1}, h_{2}, h_{3}$ subtracts the minimum value which equals the $X_{\max }-X_{\min }$. By changing the factors listed in the maximum level table, the test target replication rate also acquires maximum change and, conversely, makes minimum change.

Table 2 shows that factor $A$ has the smallest different value (1.1\%), so the level of factor $A$ changes, which has minimal impact on the replication rate. The third level of factor $A$ corresponds to the maximum average value of replication rate as $62.1 \%$, so the third level of factor $A$ is the best. Factor $B$ has the biggest different value $(34.8 \%)$, so if the level of factor $B$ changes, it has maximum impact on the replication rate; meanwhile, factor $B$ should be used as a major factor in the study. The third level of factor $B$ corresponding to the maximum average value of replication rate is $78.7 \%$, so the third level of factor $B$ is the best. The different value of factor 
TABLE 2: The coupling test analysis table of three factors $A, B$, and C.

\begin{tabular}{|c|c|c|c|c|c|c|c|}
\hline & Factors & & & & & esults & \\
\hline Number & $A$ & $B$ & $C$ & & Replica & ion rat & $e / \%$ \\
\hline & & & & $N 1$ & N2 & N3 & Average \\
\hline 1 & 1 & 1 & 1 & 38.7 & 39.4 & 38.4 & 38.8 \\
\hline 2 & 1 & 2 & 2 & 61.7 & 59.6 & 59.7 & 60.3 \\
\hline 3 & 1 & 3 & 3 & 83.2 & 84.3 & 83.8 & 83.8 \\
\hline 4 & 2 & 1 & 2 & 41.3 & 42.1 & 45.7 & 43.0 \\
\hline 5 & 2 & 2 & 3 & 68.6 & 67.6 & 68.5 & 68.2 \\
\hline 6 & 2 & 3 & 1 & 73.8 & 74.6 & 73.7 & 74.0 \\
\hline 7 & 3 & 1 & 3 & 49.0 & 50.6 & 51.2 & 50.3 \\
\hline 8 & 3 & 2 & 1 & 57.3 & 57.6 & 57.4 & 57.4 \\
\hline 9 & 3 & 3 & 2 & 78.2 & 78.6 & 78.7 & 78.5 \\
\hline$H_{1}$ & 182.9 & 132.1 & 170.2 & & & & \\
\hline $\mathrm{H}_{2}$ & 185.2 & 185.9 & 181.8 & & & & \\
\hline $\mathrm{H}_{3}$ & 186.2 & 236.3 & 202.3 & & & & \\
\hline$h_{1}$ & 61.0 & 44.0 & 56.7 & & & & \\
\hline$h_{2}$ & 61.7 & 62.0 & 60.6 & & & & \\
\hline$h_{3}$ & 62.1 & 78.8 & 67.4 & & & & \\
\hline Value & 1.1 & 34.8 & 10.7 & & & & \\
\hline Rank & 3 & 1 & 2 & & & & \\
\hline Best program & $A_{3}$ & $B_{3}$ & $\mathrm{C}_{3}$ & & & & \\
\hline
\end{tabular}

$C$ is $10.7 \%$, and the third level of factor $C$ corresponds to the maximum average value of replication rate as $67.4 \%$, so the third level of factor $C$ is the best. So the three factors effects on the replication rate are ranked as $B, C, A$, and the best program is number 3 in Table 2, whose replication rate is $83.8 \%$, in which $B_{3}$ : rolling speed, the third level, and $0.5 \mathrm{rad} / \mathrm{s}, C_{3}$ : rolling temperature, the third level and $150^{\circ} \mathrm{C}$, and $A_{3}$ : rolling depth, the third level, and $0.6 \mathrm{~mm}$.

As the best program $B_{3} C_{3} A_{3}$ is not listed in the total 9 tests, the program $B_{3} C_{3} A_{3}$ is tested to verify whether we can get the best results by orthogonal experiment method, then the calculated replication rate is $87.9 \%$ by formula (15), and the replication rate is higher than what replication rate obtains in test number 3 , which shows that the best program $B_{3} C_{3} A_{3}$ by orthogonal experiment complied with the actual situation.

It has been found through the above analysis that the best replication rate is $87.9 \%$ and realized the pit-type replication of nonsmooth surface. The reason that the hotroller processing cannot achieve complete replication of pittype nonsmooth surface is that when hot-roller head is in contact with polymer coating, its temperature is higher than temperature $T_{q}$ in which the glass state transits to viscoelastic state, and polymer coating is in viscoelastic state. When the two separates, polymer coating will be cooling with natural surrounding environment temperature, and polymeric materials will appear the rebound phenomena, which leads pit structure to tiny deformation, changing pit morphology, and then reducing the replication rate.

\section{Conclusions}

The structural model of walking modules and processing module for the pipe inner-wall machining robot is established and their work process is analyzed. What is more, kinetic equations of hot-rolling process are also established, and the relationships between angular velocity, output torque, and rolling reaction force of rolling cutter are obtained. In addition, surface morphology of the roller-head is designed, and the principle of selecting roller-head power is given.

The experimental prototype of pipe inner-wall machining robot is built, and the method of coating the polymer pipe inner-wall is given, and then the conditions of hot-rolling processing are designed. Experimental method is used to analyze hot-rolling effects under different process parameters. Meanwhile, it is also verified that the rolling temperature will be elevated and the speed of rolling will be decreased. It shows that both can improve the pit structure replication rates of the polymer coating surface, and the rolling depth has little effect on replication rates.

The effect of hot-rolling process parameters is analyzed on pit morphology through orthogonal method. According to effect of replication rate on various factors, the ranked order is obtained: rolling speed, rolling temperature, and rolling depth. The best level program is $B_{3} C_{3} A_{3}$ with replication rate of $87.9 \%$. The reason that hot-roller processing cannot achieve the copy of the pit-type nonsmooth surface completely is the viscoelastic state of polymer coating. When hot-rolling-head separates from polymer coating, polymer coating encountered rebound phenomena, which leads pit structure to tiny deformation and reduces the replication rate.

\section{Conflict of Interests}

The authors declare that there is no conflict of interests regarding the publication of this paper.

\section{Acknowledgment}

The work was supported by the Zhejiang Provincial Natural Science Foundation of China (Grant no. LQ15E050005).

\section{References}

[1] H. Tan, A. Gilbertson, and S. Y. Chou, "Roller nanoimprint lithography," Journal of Vacuum Science and Technology B, vol. 16, no. 6, pp. 3926-3928, 1998.

[2] S.-W. Youn, M. Ogiwara, H. Goto, M. Takahashi, and R. Maeda, "Prototype development of a roller imprint system and its application to large area polymer replication for a microstructured optical device," Journal of Materials Processing Technology, vol. 202, no. 1-3, pp. 76-85, 2008.

[3] S. H. Ahn and L. J. Guo, "Large-area roll-to-roll and roll-toplate nanoimprint lithography: a step toward high-throughput application of continuous nanoimprinting," ACS Nano, vol. 3, no. 8, pp. 2304-2310, 2009.

[4] M. G. Lee, S. H. Lan, S. H. Lee, H. J. Lee, J. Ni, and Y. W. Sung, "Development of roll-to-flat thermal imprinting equipment and experimental study of large area pattern replication on polymer 
substrate," Journal of the Korean Society of Manufacturing Technology Engineers, vol. 18, no. 3, pp. 307-314, 2009.

[5] J. Hou, Y. Chen, Y. S. Li, X. Weng, H. Li, and C. Pereira, “32.3: Reliability and performance of flexible electrophoretic displays by roll-to-roll manufacturing processes," SID Symposium Digest of Technical Papers, vol. 35, no. 1, pp. 1066-1069, 2004.

[6] Y.-H. Yeh, C.-C. Cheng, B. C.-M. Lai, C.-M. Leu, and Y.-L. Tseng, "Flexible hybrid substrates of roll-to-roll manufacturing for flexible display application," Journal of the Society for Information Display, vol. 21, no. 1, pp. 34-40, 2013.

[7] X.-J. Shen, L.-W. Pan, and L. W. Lin, "Microplastic embossing process: experimental and theoretical characterizations," Sensors and Actuators A: Physical, vol. 97-98, no. 1-2, pp. 428-433, 2002.

[8] X. C. Shan, R. Maeda, T. Ikehara, H. Mekaru, and T. Hattori, "Fabrication of X-ray masks and applications for optical switch molding," Sensors and Actuators A: Physical, vol. 108, no. 1-3, pp. 224-229, 2003.

[9] X. C. Shan, T. Ikehara, Y. Murakoshi, and R. Maeda, "Applications of micro hot embossing for optical switch formation," Sensors and Actuators A: Physical, vol. 119, no. 2, pp. 433-440, 2005.

[10] R. C. Liang, J. Hou, H. M. Zang, J. Chung, and S. Tseng, "Microcup ${ }^{\circledR}$ displays: electronic paper by roll-to-roll manufacturing processes," Journal of the Society for Information Display, vol. 11, no. 4, pp. 621-628, 2003.

[11] C.-Y. Chang, S.-Y. Yang, L.-S. Huang, and J.-H. Chang, "Fabrication of plastic microlens array using gas-assisted micro-hotembossing with a silicon mold," Infrared Physics and Technology, vol. 48, no. 2, pp. 163-173, 2006.

[12] J. L. Charest, L. E. Bryant, A. J. Garcia, and W. P. King, "Hot embossing for micropatterned cell substrates," Biomaterials, vol. 25, no. 19, pp. 4767-4775, 2004.

[13] K.-J. Byeon, K.-Y. Yang, and H. Lee, “Thermal imprint lithography using sub-micron sized nickel template coated with thin $\mathrm{SiO}_{2}$ layer," Microelectronic Engineering, vol. 84, no. 5-8, pp. 1003-1006, 2007.

[14] H. Mekaru, H. Goto, and M. Takahashi, "Development of ultrasonic micro hot embossing technology," Microelectronic Engineering, vol. 84, no. 5-8, pp. 1282-1287, 2007.

[15] G.-B. Lee, S.-H. Chen, G.-R. Huang, W.-C. Sung, and Y.-H. Lin, "Microfabricated plastic chips by hot embossing methods and their applications for DNA separation and detection," Sensors and Actuators B: Chemical, vol. 75, no. 1-2, pp. 142-148, 2001.

[16] J.-H. Chang and S.-Y. Yang, "Gas pressurized hot embossing for transcription of micro-features," Microsystem Technologies, vol. 10, no. 1, pp. 76-80, 2003.

[17] X. J. Wang, H. M. Zang, and P. Li, “48.5: roll-to-roll manufacturing process for full color electrophoretic film," SID Symposium Digest of Technical Papers, vol. 37, no. 1, pp. 1587-1589, 2006.

[18] J. M. Li, C. Liu, and J. Peng, "Effect of hot embossing process parameters on polymer flow and microchannel accuracy produced without vacuum," Journal of Materials Processing Technology, vol. 207, no. 1-3, pp. 163-171, 2008.

[19] S. Lan, H.-J. Lee, S.-H. Lee et al., "Experimental and numerical study on the viscoelastic property of polycarbonate near glass transition temperature for micro thermal imprint process," Materials and Design, vol. 30, no. 9, pp. 3879-3884, 2009.

[20] S. H. Lan, H.-J. Lee, E. Kim et al., "A parameter study on the micro hot-embossing process of glassy polymer for pattern replication," Microelectronic Engineering, vol. 86, no. 12, pp. 2369-2374, 2009.
[21] S. Lan, J.-H. Song, M. G. Lee, J. Ni, N. K. Lee, and H.-J. Lee, "Continuous roll-to-flat thermal imprinting process for large-area micro-pattern replication on polymer substrate," Microelectronic Engineering, vol. 87, no. 12, pp. 2596-2601, 2010.

[22] Y. Q. Gu, G. Zhao, W. B. Yu, J. G. Mou, and S. H. Zheng, "Modeling for a non-smooth surface processing robot and hot-roll technique tests," Journal of Vibration and Shock, vol. 34, no. 3, pp. 221-228, 2015.

[23] P. R. Viswanath, "Aircraft viscous drag reduction using riblets," Progress in Aerospace Sciences, vol. 38, no. 6-7, pp. 571-600, 2002.

[24] F. E. Fish, “The myth and reality of Gray's paradox: implication of dolphin drag reduction for technology," Bioinspiration and Biomimetics, vol. 1, no. 2, pp. R17-R25, 2006.

[25] Y. Q. Gu, G. Zhao, J. X. Zheng et al., "Drag reduction characteristics on jetting surface with jet angle-jet velocity coupling," Journal of Xian Jiaotong University, vol. 46, no. 9, pp. 71-77, 2012.

[26] Y. Q. Gu, G. Zhao, J. X. Zheng, Z. Y. Li, W. B. Liu, and F. K. Muhammad, "Experimental and numerical investigation on drag reduction of non-smooth bionic jet surface," Ocean Engineering, vol. 81, no. 1, pp. 50-57, 2014.

[27] D. W. Bechert, M. Bruse, W. Hage, and R. Meyer, "Fluid mechanics of biological surfaces and their technological application," Naturwissenschaften, vol. 87, no. 4, pp. 157-171, 2000.

[28] J.-C. Huang and M. W. Johnson, "Boundary layer receptivity measurements on compliant surfaces," International Journal of Heat and Fluid Flow, vol. 29, no. 2, pp. 495-503, 2008.

[29] Y.-Q. Gu, G. Zhao, H. Liu et al., "Characteristics of drag reduction of bionic dimpled surface of shell rubber ring of aerodynamic extinguishing cannon," Journal of Jilin University (Engineering and Technology Edition), vol. 43, no. 4, pp. 983990, 2013.

[30] Y.-Q. Gu, G. Zhao, H. Liu et al., "Characteristics of seal shell body's rubber ring with bionic dimpled surfaces of aerodynamic extinguishing cannon," Journal of Central South University, vol. 20, no. 11, pp. 3065-3076, 2013.

[31] Y.-Q. Gu, J.-G. Mou, D.-S. Dai et al., "Characteristics on drag reduction of bionic jet surface based on earthworm's back orifice jet," Acta Physica Sinica, vol. 64, no. 2, Article ID 024701, 2015.

[32] X. Han, D. Y. Zhang, X. Li, and Y. Li, "Bio-replicated forming of the biomimetic drag-reducing surfaces in large area based on shark skin," Chinese Science Bulletin, vol. 53, no. 10, pp. 15871592, 2008.

[33] X. Han and D. Y. Zhang, "Study on the micro-replication of shark skin," Science in China, Series E: Technological Sciences, vol. 51, no. 7, pp. 890-896, 2008.

[34] L. Fedrizzi, F. J. Rodriguez, S. Rossi, F. Deflorian, and R. Di Maggio, "The use of electrochemical techniques to study the corrosion behaviour of organic coatings on steel pretreated with sol-gel zirconia films," Electrochimica Acta, vol. 46, no. 24-25, pp. 3715-3724, 2001.

[35] M. Doherty and J. M. Sykes, "Micro-cells beneath organic lacquers: a study using scanning Kelvin probe and scanning acoustic microscopy," Corrosion Science, vol. 46, no. 5, pp. 1265$1289,2004$. 


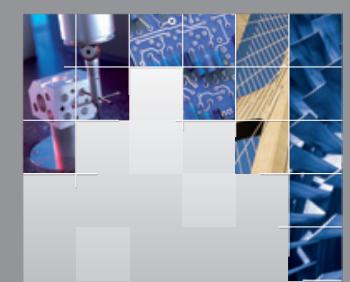

\section{Enfincering}
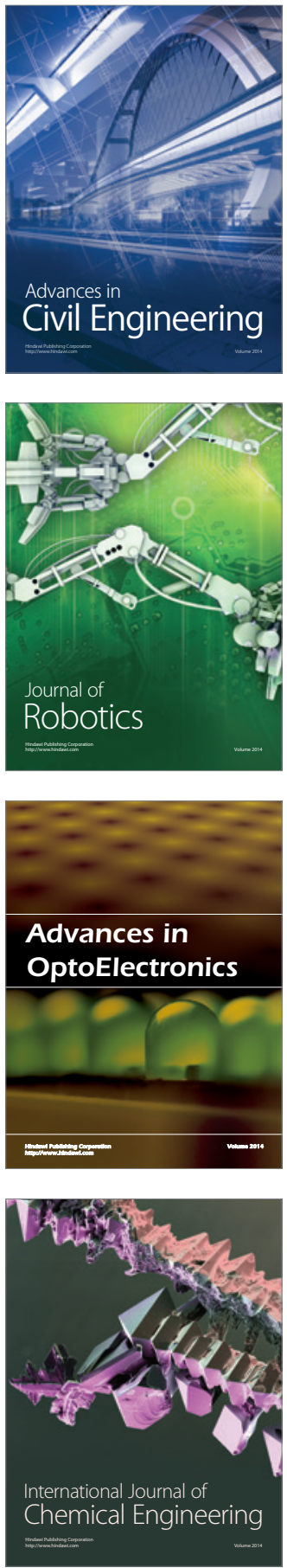

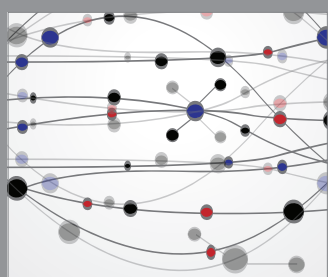

The Scientific World Journal

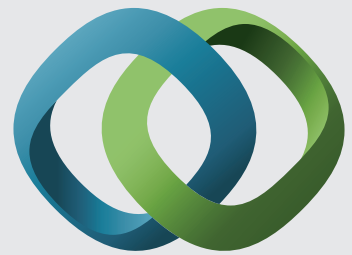

\section{Hindawi}

Submit your manuscripts at

http://www.hindawi.com
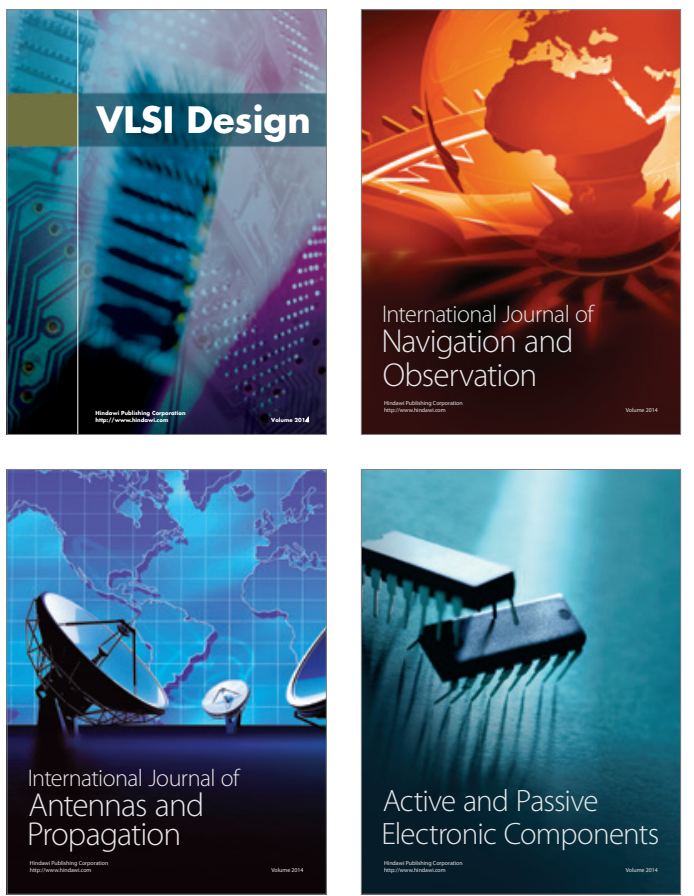
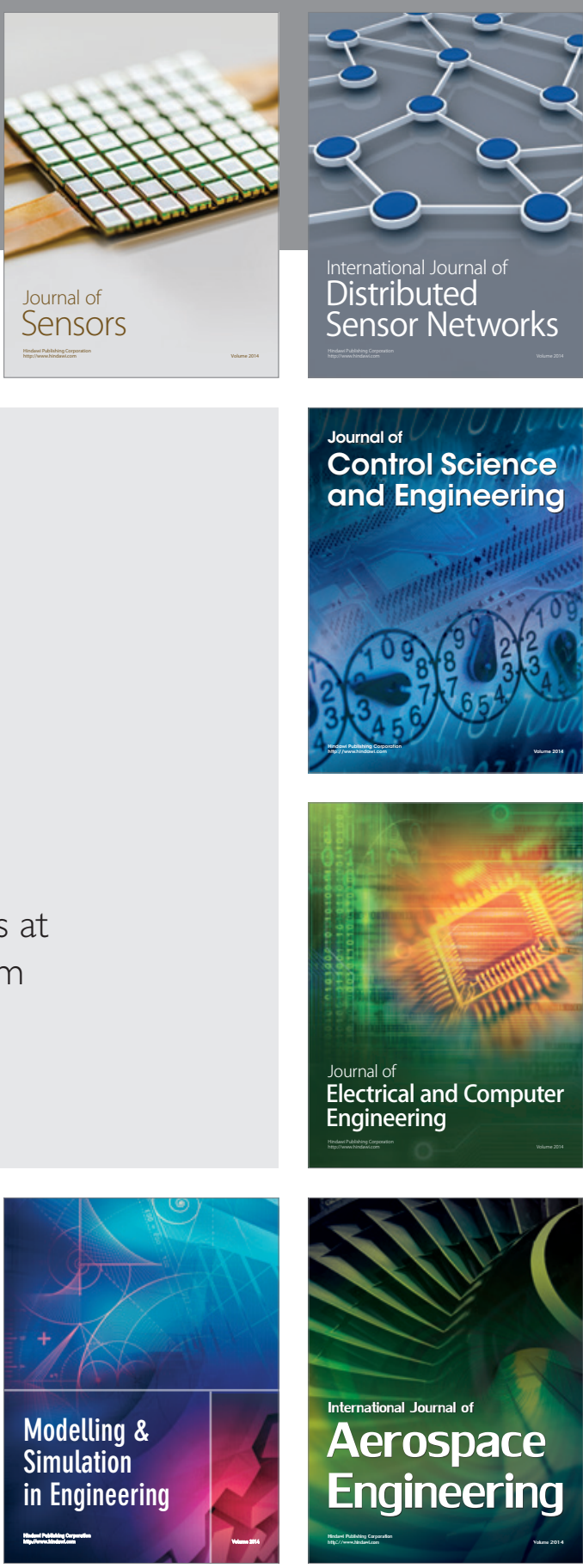

International Journal of

Distributed

Sensor Networks

Journal of

Control Science

and Engineering
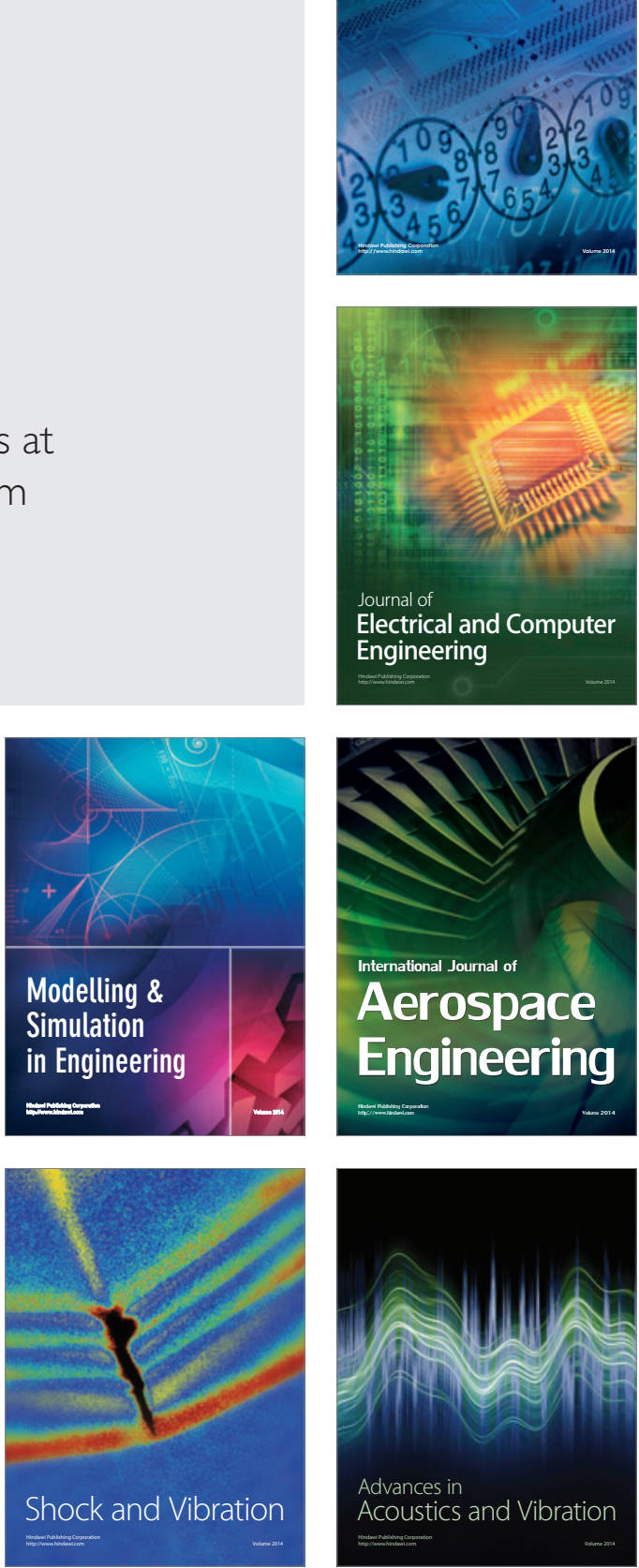